\title{
Design and Dynamic Characteristics Study on structure of MIMU Damping System
}

\author{
Tong Zhou, Meiping Wu, Bing Luo, Changfeng Chen \\ College of Mechatronics Engineering and Automation, \\ National University of Defense Technology, Changsha 410073, China \\ Zhou1011wei@163.com
}

Keywords: damping system; finite element; modal analysis; random vibration analysis

\begin{abstract}
The accuracy of a micro inertial navigation system is greatly influenced by the complex vibration environment of the vehicle, an embedded vibration attenuation system structure is designedand its rigidity is checked. The correctness of this structure design is verified through the modal analysis and frequency response analysis using the finite element analysis software. Finally, based on the frequency response analysis, the model of damping system is carried out the random vibration response analysis, and an acceleration power spectral densitycurve of the structure under the vibration environment is obtained, which provides a reliable basis for the structure design.
\end{abstract}

\section{Introduction}

The inertial navigation system is an autonomous navigation system in real sense, which can provide the navigation information of the vehicle autonomously through the inertial unit, such as acceleration, velocity, attitude and etc. without the intervention of outside information[1]. With the development of MEMS technology and the emergence of micro accelerometer and gyroscope, MIMU also appeared.The inertial unit in the micro inertial navigation system is directly fixed with the vehicle, while the vehicle is excited by many factors under the flight conditions, such as the vibration of the engine, the pulsation pressure of the turbulent boundary layer and etc. which will comprehensively produce a broadband and a large amplitude vibration excitation[2, 3]. The random error of the micro inertial unit is increased due to this vibration of the vehicle making the attitude angle error be increased, which can influence the navigation accuracy so as to reduce the measurement accuracy of the whole micro inertial navigation system greatly[4]. Therefore, in order to reduce the influence of vibration on the navigation accuracy of the system and improve the navigation accuracy of the micro inertial navigation system, it is necessary to study the damping structure and dynamic characteristics of MIMU system.

\section{MIMU embedded framestructural design}

According to the requirement of the inertial navigation principle, the data acquisition circuit board, the core processing board, the interface board, the three-piece MEMS gyroscope circuit board and the micromechanical accelerometer board are required to achieve the orthogonal installation. Based on this requirement and the minimization goal of the MIMU components, an embedded MIMU frame structure is designed, as shown in Figure 1. The three sides of the MIMU frame are embedded micro-gyroscope board and micromechanical accelerometer board, and the other three sides are respectively installed the data acquisition board, the core processing board and the interface board. 


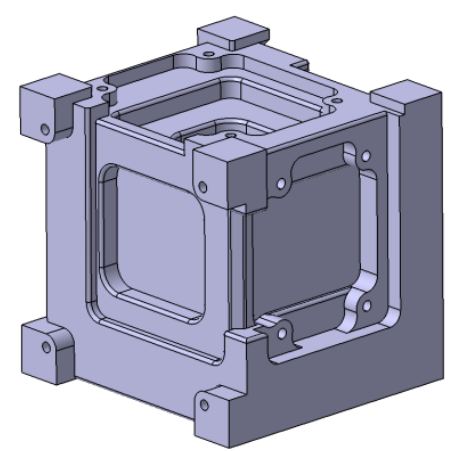

Fig.1 Three-dimensional model of IMU framework

In order to verify whether its strength meets the requirements, the rigidity of the frame is checked. The resonance points of the frame under the loading condition together with the stress distribution under the resonance status are checked by using the finite element simulation. Sincethere is no fixed connection point for the IMU frame, the simulation is carried out by the free constraint mode. As shown in Figure 2, it can be found that the first 6 order resonant modes arefree motion modes for the rigid body with the frequencies of 0 or close to 0 and that at the resonance pointsof $6193 \mathrm{~Hz}$ much higher than $500 \mathrm{~Hz}$ (the main power spectral density band under the mechanical vibration environment), which fully meets the structural strength and rigidity requirements.

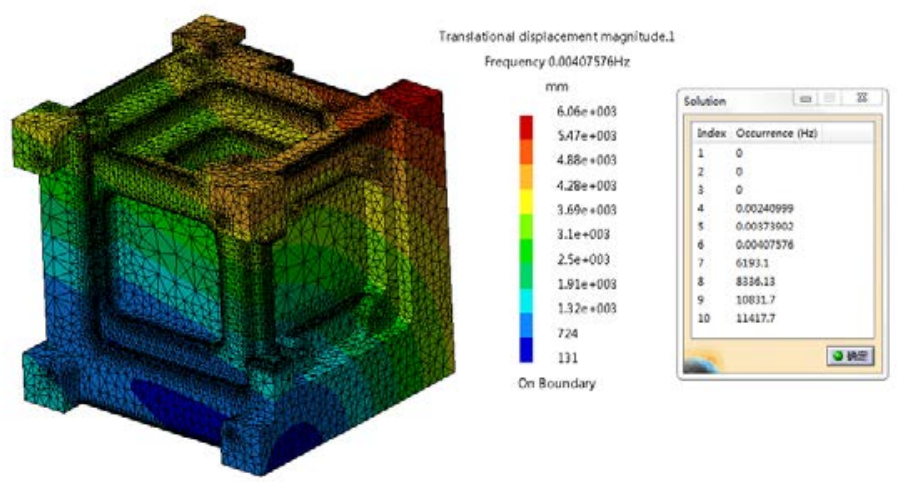

Fig.2 IMU framework resonance stress nephogram

\section{The design of vibration damping of MIMU system}

The gyroscope of MIMU is a kind of vibratory silicon micro-machined gyroscopesbased on the Coriolis Effect, and its working modes are drive-mode and detect-mode.The external vibration will affect its vibration modesbecause of its special vibrational structure, which will cause the changes of drive-mode, and then the output error is produced.In order to control the source of vibration, the design of vibration damping is necessary.

The damper scheme and finite element modeling.In the classical vibration theory, the eccentricity of the rigid body and the asymmetry of support waywill cause the motion coupling of the six degrees of freedom.Therefore, it is very important to design the damping systemscheme to meet the requirements of the application. At present, six kinds of vibration isolation pattern are mostly used in the structural design of inertial navigation systems [5], and the article uses the basic model of the eight point vibration absorption. The vibration damping structure model is imported into MSC.Patran, and each part is meshed respectively, as shown in Figure 3. 


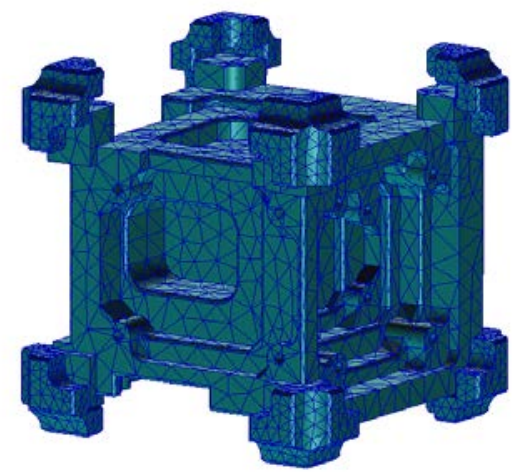

Fig.3 Finite element model of MIMU damping structure

Mode analysis.The pre-processing and post-processing of the MIMU dampingstructure by using the finite element software Patran and Nastran are made, then we get the MIMU's 20-order damping system modal frequencies and mode shapes [6].The correspondingorders and frequenciesare shown in Table 1. From the analysis, the frequency of vibration damping is greatly reducedcompared to the previous, and the frequency pointsare concentrated near the $150 \mathrm{HZ}, 170 \mathrm{HZ}$ and $250 \mathrm{HZ}$, meeting the requirement that the MIMU system vibration modal frequency is not dispersed. Figure 4 shows the modal formation diagram of the MIMU damping system. Its first 6 order formations mainly show the framework deformation, 7 20 order formations are mainly local deformation of damping rubbers.

Table 1Modal analysis result of MIMU damping system

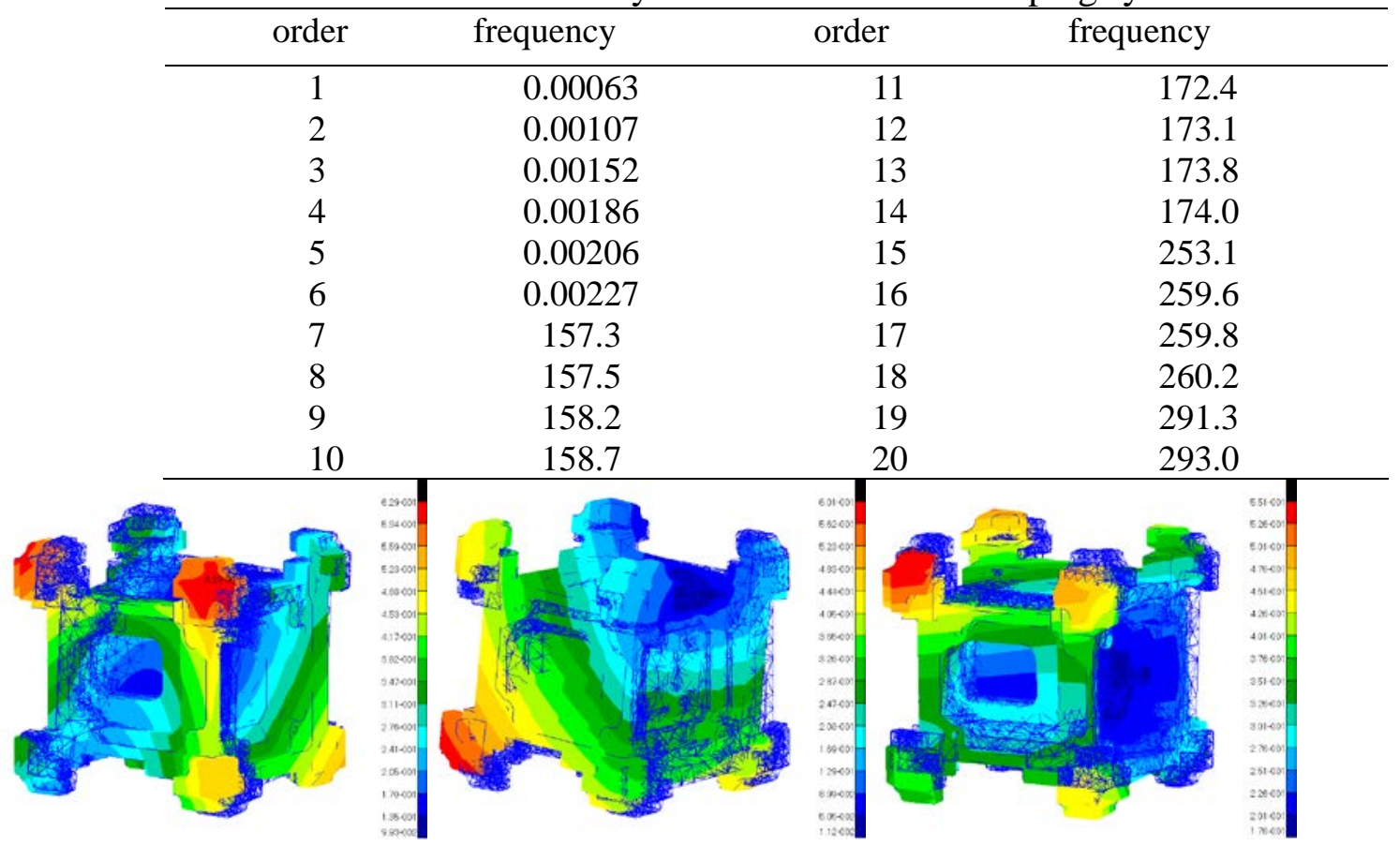

(a) The first order formation(b)Thesecondorder formation(c)The third order formation
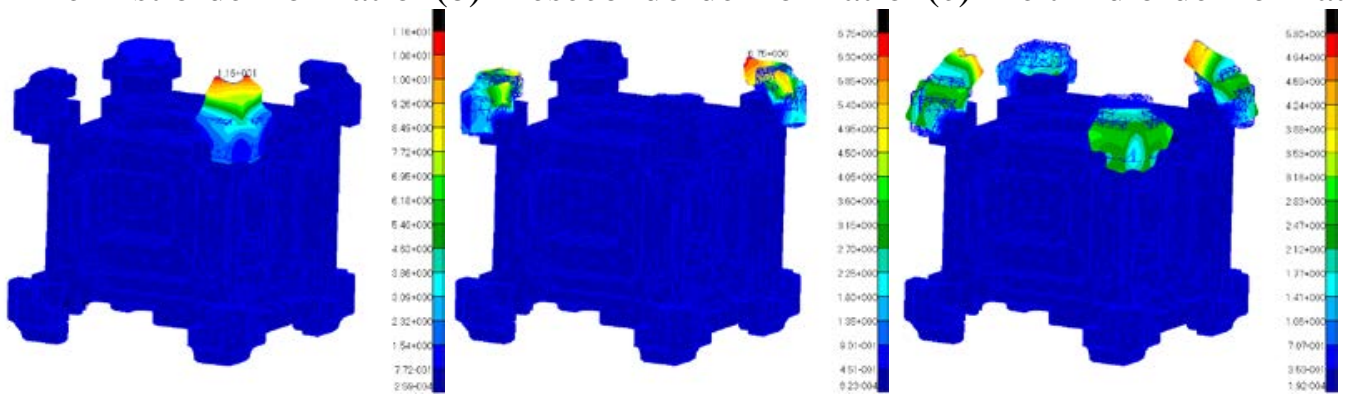

(d)The seventh order formation (e)The 12thorder formation(f)The 19th order formation

Fig.4 The modal formation diagram of the MIMU damping system 


\section{Frequency response analysis of MIMU damping system}

The frequency response analysis of the finite element model of the MIMU damping system is carried out under the periodic load.The load is transferred to the model by establishing the MPC unit.The excitation frequency range is $1.0 \sim 1000.0 \mathrm{~Hz}$, and the structural damping coefficient is 0.03 ,then use modal superposition method to analyze. In this paper, we select three nodes 5605 , 10381,49280 on three boards as the response points, and the displacement response is shown in Figure 5.It can be seen from Fig.5 that the displacements of the chosen three nodes are peak at frequency around $290 \mathrm{~Hz}$, and the peak frequency avoids thevibration frequency of the micro-gyroscope, thus there is no resonance phenomenon, which is further proved the correctness of the model.

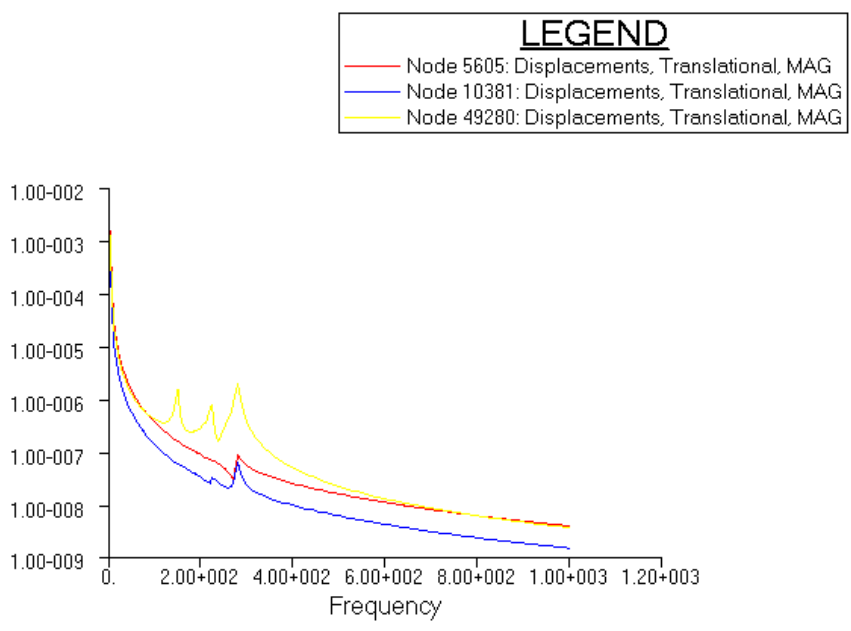

Fig.5 Displacement response diagram of three nodes

\section{Random vibration response analysis}

The random vibration responseanalysis is made by using the Analysis Random command in Patranbased on the frequency response analysis. The bottom of the foundation of the MIMU damping system is fixed, and the Z-axis direction is the most vulnerable to external vibration interference. Therefore, this paper adds a random vibration input in the Z-axis direction. The applied power spectral density(PSD) ofrandom vibration is shown in table 2.

Table 2 PSDof random vibration

\begin{tabular}{cc}
\hline Frequency range/Hz & $\mathrm{PSD} /(\mathrm{g} 2 / \mathrm{Hz})$ \\
\hline $20 \sim 170$ & 0.01 \\
$170 \sim 500$ & $+3 \mathrm{~dB} /$ oct \\
$500 \sim 1000$ & 0.03 \\
$1000 \sim 2000$ & $-4 \mathrm{~dB} /$ oct
\end{tabular}

After the random vibration response analysis of the vibration damping structure,the same three nodes in the frequency response are chosen as the response points.The PSD response curves of three nodes as shown in figure 6 . From the figure, the acceleration power spectrum curves of the three plates aresimilar. The responses are concentrated in the vicinity of the peak, and there are changes at the natural frequency, which shows that the natural frequency of the system has an important influence on the random vibration response. 


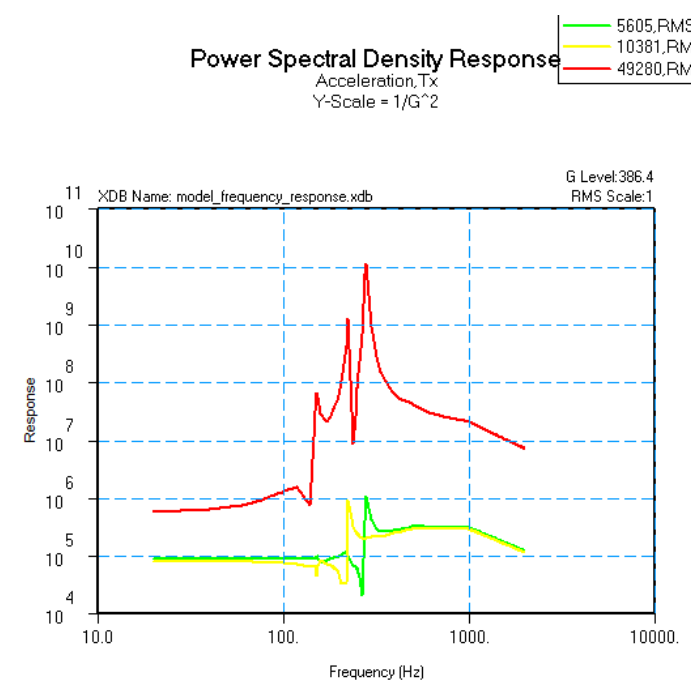

Fig.6 The PSD response curves of three nodes

\section{Conclusion}

In this article, the rigidity of the MIMU embedded structure frame is checked by finite element simulation, and avibration attenuation system structure of MIMU is designed, the finite element model is established by using MSC series of finite element software, then the dynamic characteristics of the model are analyzed, including modal analysis, frequency response analysis and random vibration analysis. All these verify the correctness of the design of the damping system, and provide a theoretical basis for the structural design.

\section{Reference}

[1] Lahham J I, Wigent D J, Coleman A L. Tuned support structure-borne noise reduction ofinertial navigation with dithered ring laser gyros(RLG)// Proceedings of the IEEE Position Location and Navigation Symposium, 2000: 418-428.

[2] Rao S S. Mechanical vibrations. 5th Ed. London: Pearson Prentice Hall, 2010: 281-284.

[3] TUO Zhou-hui, HU De-wen, LI Ru-hua, et al. Damping design of strap-down inertial navigation system. Journal of Chinese Inertial Technology, 2009, 17(6): 648-650.

[4] Geiger W, Bartholomeyczik J , Breng U, et al . MEMS IMU for AHRS applicationsIEEE/IONPosition Location and NavigationSymposium, 2008:225-231.

[5] YAO Jian-jun. Contrast of different vibration isolation patterns used in strap-down inertial navigation system. Structure \& Environment Engineering, 2009, 36(2): 19-27.

[6] LIU Jian-feng, DING Chuan-hong, HAO Chun-zhao, SONG Geng-yue.Modal analysis of IMU structure based on B-spline wavelet finite element. Systems Engineering and Electronics, 2007(11): 1958-1961. 